\title{
EMDA mitomycin before TURBT is the best treatment for non-muscle-invasive disease
}

Bladder cancer is the seventh most common cancer in men, with nearly 400,000 cases diagnosed per year. However, despite the use of transurethral resection of the bladder tumor (TURBT), a large number of patients will relapse, many of whom will progress to muscleinvasive disease. Postoperative intravesical instillation of mitomycin is currently recommended in clinical guidelines for treatment of bladder cancer, and is thought to reduce recurrence by preventing seeding of tumor cells exfoliated during the TURBT procedure.

A team from Italy has investigated the efficacy of combination therapy using TURBT with preoperative or postoperative mitomycin, publishing their findings in the Lancet Oncology. Mitomycin is a commonly used chemotherapy drug in the treatment of bladder cancer, and can be administered using passive diffusion (PD) or electromotive drug administration (EMDA) techniques. "EMDA describes accelerated drug transport under the influence of an electric field," explains Savino Di Stasi, from Rome's Tor Vergata University, who led the team. "Mitomycin is nonionized within the tolerable physiological range and its electromotive mode of delivery is by electroosmosis. In laboratory and clinical studies, intravesical EMDA increased mitomycin bladder uptake, improving clinical efficacy in high-risk non-muscle-invasive bladder cancer, without relevant local and systemic toxicity." However, EMDA mitomycin is not recommended after TURBT, owing to the increased risk of mechanical trauma and urothelial injury.

The multicenter, randomized parallelgroup study enrolled patients at three centers in Italy between 1994 and 2003. Eligible patients had primary pTa and pT1 urothelial carcinoma and were randomly assigned to one of three treatment groups: TURBT alone, immediate passive diffusion (PD) mitomycin after TURBT, or immediate EMDA mitomycin before TURBT. Patients who were randomized to postoperative $\mathrm{PD}$ mitomycin received $40 \mathrm{mg}$ mitomycin within $6 \mathrm{~h}$ of completion of TURBT. Patients receiving preoperative EMDA mitomycin were infused intravesically with $40 \mathrm{mg}$ mitomycin, while a $20 \mathrm{~mA}$ pulsed current was administered externally around $30 \mathrm{~min}$ before anesthesia was given for TURBT. Recurrence rate and disease-free interval were used as primary end points.

Patients who received preoperative EMDA mitomycin had a lower recurrence rate than those who received postoperative PD mitomycin, of $38 \%$ and $59 \%$, respectively. Patients undergoing preoperative EMDA mitomycin administration also had a longer diseasefree interval, of 52 months, compared with only 16 months in those who received postoperative $\mathrm{PD}$ mitomycin and 12 months in those who had surgery alone.

Overall, intravesical EMDA mitomycin before TURBT provided significant longterm benefit in all stratification groups including intermediate-risk and highrisk patients and those with multifocal disease. As Di Stasi summarizes: "Our findings suggest that intravesical EMDA mitomycin before TURBT ensures a higher and deeper bladder target tissue concentration able to destroy or inhibit the implantation of viable cancer cells. We

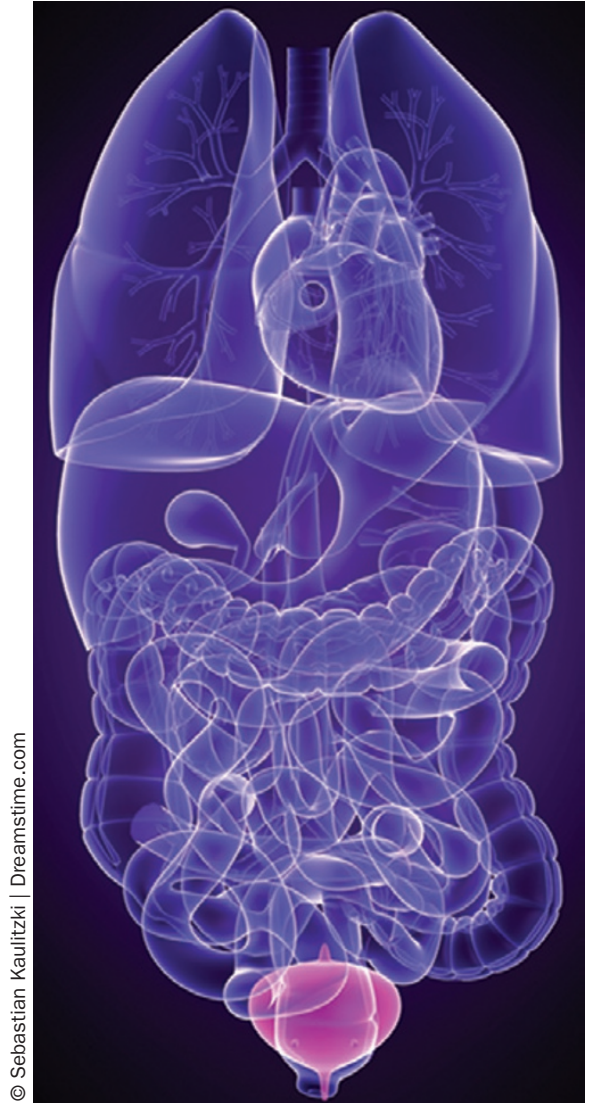

hope that future developments will enable intravesical EMDA mitomycin to be added to standardized treatment protocols."

Annette Fenner

Original article Di Stasi, S. M. et al. Electromotive instillation of mitomycin immediately before transurethral resection for patients with primary urothelial non-muscle invasive bladder cancer: a randomised controlled trial. Lancet Oncol. doi:10.1016/S1470-2045(11)70190-5 